\title{
Study on The Symptoms and Esophageal Motility Characteristics of Gastroesophageal Reflux Disease Patients with Obesity
}

\section{Yifan Yang}

Laparoscopic Surgery Department, First Affiliated Hospital of Dalian Medical University

\section{Attique Ur Rehman}

Neurogastroenterology and Motility Center of China-US Cooperation, Second Gastroenterology Department, First Affiliated Hospital of Dalian Medical University

\section{Lixia Wang}

Neurogastroenterology and Motility Center of China-US Cooperation, Second Gastroenterology Department, First Affiliated Hospital of Dalian Medical University

\section{Dong Yang}

Neurogastroenterology and Motility Center of China-US Cooperation, Second Gastroenterology Department, First Affiliated Hospital of Dalian Medical University

\section{Zhifeng Zhang}

Nuerogastroenterology and Motility Center of China-US Cooperation, Second Gastroenterology Department, First Affiliated Hospital of Dalian Medical University

\section{Ping Zhang}

Nuerogastroenterology and Motility Center of China-US Cooperation, Second Gastroenterology Department, First Affiliated Hospital of Dalian Medical University

\section{Liping Su}

Nuerogastorenterology and Motility Center of China-US Cooperation, Second Gastroenterology Department, First Affiliated Hospital of Dalian Medical University

\section{Shi Jin}

Laparoscopic Surgery Department, First Affiliated Hospital of Dalian Medical University

\section{Zhijun Duan ( $\nabla$ cathydoctor@sina.com )}

department of gastroenterology, the first affiliated hospital of dalian medical university https://orcid.org/0000-0001-7976-4959

\section{Research article}

Keywords: esophageal motility disorder, obesity, GERD, high resolution manometry

Posted Date: October 29th, 2020 
DOI: https://doi.org/10.21203/rs.3.rs-97718/v1

License: (c) (i) This work is licensed under a Creative Commons Attribution 4.0 International License. Read Full License 


\section{Study on the Symptoms and Esophageal Motility Characteristics of Gastroesophageal Reflux Disease Patients with Obesity}

Authors: Yifan Yang, MD\&PhD, ${ }^{1}$ Attique Ur Rehman, $\mathrm{MD},{ }^{2}$ Lixia Wang, $\mathrm{MD},{ }^{2}$ Dong Yang, $\mathrm{MD},{ }^{2}$

Zhifeng Zhang, MD\&PhD, ${ }^{2}$ Ping Zhang, BD,${ }^{2}$ Liping Su, BD, ${ }^{2}$ Shi Jin, MD\&PhD, ${ }^{1}$ Zhijun Duan , $\mathrm{MD} \& \mathrm{PhD}^{2}$

From the ${ }^{1}$ Laparoscopic Surgery Department, First Affiliated Hospital of Dalian Medical University, Dalian, China; and ${ }^{2}$ Neurogastroenterology and Motility Center of China-US Cooperation, Second Gastroenterology Department, First Affiliated Hospital of Dalian Medical University, Dalian, China.

Address correspondence to: Zhijun Duan, $\mathrm{MD} \& \mathrm{PhD}$, Neurogastroenterology and Motility Center of China-US Cooperation, Second Gastroenterology Department, First Affiliated Hospital of Dalian Medical University, Dalian 116011, China (E-mail: cathydoctor@sina.com, Tel/Fax: 86-411-83635963).

Conflicts of Interest and Source of Funding: The authors declare that they have nothing to disclose. 


\section{ABSTRACT}

Background: Previous studies have shown that obesity affects the occurrence and development of GERD, GERD is also one of the many complications of obesity. However, few prior studies have evaluated the symptoms and esophageal motility characteristics of GERD patients with obesity. The purpose of this study was to assess the symptoms and esophageal motility characteristics of gastroesophageal reflux disease (GERD) patients with obesity and the relationship between symptoms and esophageal motility results.

Methods: We conducted a retrospective study. Patients were divided into four groups according to whether diagnosed with GERD and obesity: obese GERD group $(\mathrm{N}=25)$, obese non-GERD group $(\mathrm{N}=21)$, non-obese GERD group $(\mathrm{N}=29)$, non-obese non-GERD group $(\mathrm{N}=22)$. High resolution manometry (HRM) was performed to assess esophageal motility. Gastroesophageal reflux symptoms were in accordance with the GERD questionnaire (GerdQ).

Results: a) The main symptoms of obese GERD group were: reflux, upper stomach pain, nausea; in the non-obese GERD group were: reflux, heartburn and taking additional medication. b) The LES resting pressure in obese GERD group was lower than in non-obese non-GERD group, non-obese GERD group and obese non-GERD group $(P<0.05)$; The EGJ separation distance in obese GERD group 
was larger compared with non-obese non-GERD group, non-obese GERD group and obese non-GERD group $(P<0.05)$; The length of the esophagus in obese GERD group was shorter than in non-obese non-GERD group, non-obese GERD group and obese non-GERD group $(P<0.05)$; The DCI in obese GERD group was lower than in obese non-GERD group $(P<0.05)$. c) In obese GERD group, patients with reduced LES resting pressure or increased EGJ separation distance are more likely to have "reflux" symptoms.

Conclusions: Comparing with GERD patients without obesity, the symptoms, esophageal motility, esophageal motility mechanism related to the symptoms of GERD patients with obesity got different characteristics. This result provides a reference for treatment options of GERD patients with obesity.

Keywords: esophageal motility disorder, obesity, GERD, high resolution manometry 


\section{BACKGROUND}

Gastroesophageal reflux disease (GERD), defined as a gastrointestinal disease that causes symptoms or complications due to the reflux of gastroduodenal contents into the esophagus or oral cavity. ${ }^{1}$ In recent years, patients with gastroesophageal reflux disease have gradually increased, and it has become one of the most common epidemic chronic diseases affecting one third of the world's population. ${ }^{2}$ A systematic retrospective analysis suggested that the prevalence of GERD is 8.8$27.8 \%$ in Western countries and $2.5-7.8 \%$ in East Asia. ${ }^{3}$ Reflux and heartburn are the most typical symptoms of GERD. Atypical symptoms include non-cardiogenic chest pain, chronic cough, hoarse voice, sore throat, pharyngeal discomfort, and increased throat secretions. $^{4}$

Obesity is a widespread metabolic disease in the 21 st century. As time goes by, the obesity population is gradually expanding. As of 2010 , obesity has caused 3.4 million deaths and 4\% increase of Disability-Adjusted Life-Years (DALY). ${ }^{5}$ In 2016, the World Health Organization (WHO) reported that 650 million adults were diagnosed with obese, accounting for about $34 \%$ of the total overweight population. ${ }^{6}$ A survey of 192,000 people in the same year showed that according to current trends, it is estimated that by $2025,18 \%$ of men and $21 \%$ of women will be obese, and $6 \%$ of men and $9 \%$ of women will become severely obese. ${ }^{7}$ Body Mass Index (BMI) is an index that combines height and weight to determine whether the 
body is obese or not and its degree. BMI $\left(\mathrm{kg} / \mathrm{m}^{2}\right)$ was calculated as body weights (kg) divided by height squared $\left(\mathrm{m}^{2}\right)$; Chinese men and women with a BMI larger than 28 would be regarded as obese. ${ }^{8}$ Obesity can affect the functions of multiple organs of the body, leading to complications such as cardiovascular, metabolism, lung, musculoskeletal, urogenital, central nervous system, cancer and psychological issues. ${ }^{9}$

In recent years, there have been a large number of reports on the risk factors that affect the severity of GERD. Studies have shown that GERD is one of many complications caused by obesity. ${ }^{10}$ An increase in BMI can lead to an increase in the prevalence of GERD and further aggravation of symptoms. When $B M I \geq 30$ $\mathrm{kg} / \mathrm{m}^{2}$, the prevalence of GERD increased from $23 \%$ to $50 \%{ }^{11}$ A study reported that about $61 \%$ of people with obesity have reflux symptoms. ${ }^{12}$ Obesity may lead to an increased incidence of Erosive Esophagitis (EE) and Barrett's Esophagus (BE). ${ }^{13} \mathrm{~A}$ retrospective analysis showed that the risk of BE for overweight and patients with obesity is 2.5 times that of normal-weight patients. Without treatment, $10 \%$ of people will be at risk for esophageal adenocarcinoma. ${ }^{14,15}$

There are also contradictions and disputes in various research conclusions. Ayazi S et al. analyzed the data of 1659 patients and the results showed that the number of GERD patients with obesity got lower low esophageal sphincter (LES) pressure was more than twice that of normal-weight GERD patients. And as the 
patient's BMI increased, the incidence of reduced LES pressure also increased. ${ }^{16}$ Another study using Multichannel Intraluminal Impedance (MII) combined with traditional manometry techniques showed that there was no significant difference in the basic LES pressure between normal-weight GERD patients and GERD patients with obesity. ${ }^{17}$ Therefore, whether there is an exact correlation between obesity and GERD, and whether abnormal esophageal motility is the mechanism of obesity affecting GERD needs to be further explored. At present, there are few reports about the gastroesophageal reflux symptoms and esophageal dynamics in GERD patients with obesity.

In this study, the GerdQ and high resolution manometry (HRM) data of obese GERD patients were compared and analyzed with non-obese GERD, obese non-GERD patients and non-obese non-GERD. This study is aimed at exploring the symptoms and esophageal dynamics characteristics of obese GERD patients, and the relationship between the two. It is also expected that these research results will provide important references for the mechanism of GERD in patients with obesity and individualized comprehensive treatment.

\section{METHODS}

\section{Study subjects}


The Neurogastrointestinal Motility Center of the Second Department of Gastroenterology in First Affiliated Hospital of Dalian Medical University admitted a large number of patients with GERD, and the Department of Laparoscopic Surgery carried out anti-reflux and weight loss surgeries, and admitted patients with obesity and GERD patients with obesity, which provided the conditions for this study.

GERD patients were consecutively selected from those referring to the outpatient and inpatient unit of the Second Department of Gastroenterology and Department of Laparoscopic Surgery in First Affiliated Hospital of Dalian Medical University of China between November 6, 2018 and November 6, 2019 as the experimental group. And those non-GERD patients were selected who visited the Second Department of Gastroenterology and Department of Laparoscopic Surgery during the same period as the control group. All cases were performed using high resolution manometry and filled out the GerdQ in the Neurogastrointestinal Motility Center, and the department doctors recorded symptoms. If patients had extra-esophageal symptoms, they were required to conduct a systematic examination in the relevant department to exclude other systemic diseases before they could be included in the group. The participants were further divided into groups according to whether they were diagnosed with GERD and obesity: obese GERD group (O-G group, $\mathrm{n}=25)$, obese non-GERD group (O-NG group, $\mathrm{n}=21$ ), 
non-obese GERD group (NO-G group, $\mathrm{n}=29$ ) and non-obese non-GERD group (NO-NG group, $\mathrm{n}=22$ ). The GERD diagnostic criteria: 24-hour dynamic $\mathrm{pH}$ monitoring results reflux index (DeMeester score) $\geq 14.72$ or under endoscopy Esophagitis (erosive esophagitis, EE) grades C and D. ${ }^{18}$ Diagnostic criteria of obesity: All selected cases meet the diagnostic criteria for adult obesity in China: men and women with a BMI larger than 28 would be regarded as obese. ${ }^{8}$

This study has been approved by the hospital ethics committee, clinical trial number: PJ-KS-KY-2018-69(X), and all participants signed an informed consent form, experimental investigation was performed following all the guidelines for experimental investigation with human subjects required by the hospital. Patients in all four groups were asked to stop using proton pump inhibitor (Proton Pump Inhibitors, PPI), histamine $\mathrm{H}_{2}$ receptor antagonist (Histamine 2 Receptor Agonists, $\mathrm{H}_{2} \mathrm{RA}$ ), drugs that may affect esophageal motility, sedatives and antidepressants 2 weeks before the test.

\section{Inclusion and exclusion criteria}

General Inclusion criteria: (1) age between 18-75 years old, no gender limit; (2) voluntarily join the study; (3) no esophageal motility disorder based on histopathology, within the past 2 weeks no medication history, no positive signs of inflammation, and no organic disease. 
Inclusion and exclusion criteria of obese GERD group (O-G group) (N=25)

Inclusion criteria: patients meet the inclusion criteria of general selection and diagnostic criteria of GERD and obesity at the same time.

Exclusion criteria: (1) patients with a history of upper gastrointestinal surgery; (2) patients with other organic diseases of the upper digestive tract such as ulcer disease, pyloric obstruction, gastric cancer, etc.; (3) patients with any precancerous lesions, gastric and esophageal polyps and hiatal hernias who cannot tolerate highresolution esophageal pressure measurement; (4) other systemic diseases involving the digestive tract such as diabetes, autoimmune diseases, etc.; (5) severe vomiting caused by pregnancy or other reasons; (6) patients with severe heart and lung insufficiency cannot tolerate the above-mentioned examinations; (7) mental illness: schizophrenia, obsessive-compulsive disorder, moderate or above depression or anxiety, etc.; (8) those with incomplete information.

Inclusion and exclusion criteria of non-obese GERD group (NO-G group) ( $N=29)$

Inclusion criteria: patients meet the inclusion criteria of general selection, diagnostic criteria of GERD, exclude the diagnosis criteria of obesity at the same time.

Exclusion criteria: others are in agreement with those of the O-G Group.

Inclusion and exclusion criteria of obese non-GERD group (O-NG group) $(N=21)$ 
Inclusion criteria: patients meet the inclusion criteria of general selection and diagnostic criteria of obesity, exclude the diagnosis of GERD at the same time.

Exclusion criteria: others are in agreement with those of the O-G Group.

Inclusion and exclusion criteria of non-obese non-GERD group (NO-NG group) $(N=22)$

Inclusion criteria: patients meet the inclusion criteria of general selection, exclude the diagnosis of obesity and GERD at the same time.

Exclusion criteria: others are in agreement with those of the O-G Group.

\section{General information}

The medical history of all subjects was collected, including age, gender, past history, family history, medication history, smoking and drinking status. All patients' height, weight was collected and the body mass index $[\mathrm{BMI}=$ weight $(\mathrm{kg}) /$ height $\left.(\mathrm{m})^{2}\right]$ was calculated.

\section{GerdQ}

It is a symptom scale composed of heartburn, reflux, epigastric pain, nausea, sleep disturbance and the frequency of taking additional medication. Patients in the obese GERD and non-obese GERD groups are required to recall the past 7 days. Fill in the symptoms, and the sum of the points is the patient's GerdQ score, with a 
total score of $0-18$ points, then the points can be used to assess the patient's gastroesophageal reflux symptom composition ratio and symptom severity. The patient completes the questionnaire under the guidance of a professional doctor to ensure that the patient clearly understands the relevant symptoms in the form. At the same time, the instructor is prohibited from using any suggestive words on any question.

\section{Esophageal motility measurement}

In this study, high resolution manometry was performed using a 24-channel waterperfused catheter of $4.0 \mathrm{~mm}$ in diameter as used previous studies (Ningbo Maida Medical Device Inc., Ningbo, China). ${ }^{19}$ The patient was required fasting for 12 hours and stopping to drink for 6 hours prior to HRM to avoid vomiting and aspiration. Before the pressure test starts, the pressure chamber of the system was used to calibrate the pressure of the 24 pressure detection channels. Before preparing to insert the pressure measuring catheter, the patient was asked whether he has a history of nasal surgery and other operations, and check whether the nasal cavity is unobstructed by the intubation tube, and select the unobstructed side of the nasal cavity to insert the pressure measuring catheter. Instruct the patient to place the pressure measurement catheter to the appropriate depth of the esophagus after sitting in the patient's position, and observe the depth of the intubation closely. When high pressure bands appear on the upper and lower ends of the 
pressure graph on the computer system interface, the intubation depth will be reached. The pressure bands respectively indicate the upper esophageal sphincter (UES)and LES. After the patient adapts for a few minutes, slowly adjust from the sitting position to the supine position, and further adjust the catheter position to ensure that there are 2 pressure measurement channels within $2 \mathrm{~cm}$ of the lower end of the LES. Fix the pressure measurement catheter and check the pressure of the UES and LES and start to collect data. First select a stable graph without swallowing action as the resting pressure (for about 30 seconds) (Fig. 1A), and then inform each patient to swallow 10 times but only $5 \mathrm{ml}$ warm water each time. The swallowing caused by the patient's choking or non-cooperation is not included in the data statistics. After a total of 10 swallows, the data obtained is input into the computer for analysis, normal Image of esophageal contraction (Fig. 1B). The LES-related indicators (LES resting pressure, LES length), esophageal length, esophageal distal contraction integral (DCI), esophagus-gastric junction (EGJ) separation distance, were recorded.

According to the " The Chicago Classification of esophageal motility disorders ", the HRM parameters and normal values:

(1) Esophageal morphological parameters: the normal range of lower esophageal sphincter resting pressure (LESP) is $13-43 \mathrm{mmHg}$; 
(2) Esophageal body peristalsis parameters: DCI, usually used to judge contraction strength, the normal value range is $450-8000 \mathrm{mmHg} \bullet \mathrm{s}^{\bullet} \mathrm{cm}$. DCI $<100 \mathrm{mmHg} \bullet \mathrm{s}^{\bullet} \mathrm{cm}$ belongs to loss of peristaltic contraction, and DCI of $100-450 \mathrm{mmHg} \bullet \mathrm{s}^{\bullet} \mathrm{cm}$ belongs to weak peristaltic contraction.

(3) Parameters of the EGJ: EGJ separation distance is the distance between LES and crural diaphragm (CD) (Fig. 2). In the state of calm breathing, the Chicago standard divides the EGJ into three types according to the relative positional relationship between LES and CD. Type I: the relative position of LES and CD basically overlap, the distance between the two is $<1 \mathrm{~cm}$; Type II: the separation between LES and CD, the distance between the two is $>1 \mathrm{~cm}$, but $<2 \mathrm{~cm}$; Type III: the separation of LES and $\mathrm{CD}>2 \mathrm{~cm} .{ }^{20}$

\section{Statistical methods}

Graghpad prism6.0 program was used for statistical analysis. Two independent sample t test, rank sum test, $\chi 2$ test, Spearman correlation analysis were performed respectively. The measurement data conforming to the normal distribution is expressed as mean \pm standard deviation $(\mathrm{x} \pm \mathrm{SD})$, and the measurement data of nonnormal distribution is expressed as $[\mathrm{M}(\mathrm{p} 25, \mathrm{p} 75)] . P<0.05$ indicates that the difference is statistically significant.

\section{RESULTS}




\section{General data of patients}

There were 25 cases in the obese GERD group, with a BMI of $31.70(30.20,37.90)$ $\mathrm{kg} / \mathrm{m}^{2}$; there were 29 cases in the non-obese GERD group, with a BMI of 25.00 $(22.65,26.75) \mathrm{kg} / \mathrm{m}^{2}$; obese non-GERD patients included 21 cases, BMI was 31.60 $(29.65,36.00) \mathrm{kg} / \mathrm{m}^{2}$; There were 22 cases in the non-obese non-GERD group, with a BMI of $25.60(22.60,27.28) \mathrm{kg} / \mathrm{m}^{2}$. Compared with the four groups of patients in terms of age and gender ratio, the differences were not statistically significant $(P>0.05)$, and they were comparable (Table 1).

TABLE 1. Comparison of general information of the four groups of patients

\begin{tabular}{lllll}
\hline & O-G group & NO-G group & O-NG group & NO-NG group \\
\hline Number of cases & 25 & 29 & 21 & 22 \\
Age $($ years $)$ & $52.76 \pm 12.23$ & $53.76 \pm 12.10$ & $46.86 \pm 12.50$ & $50.64 \pm 11.89$ \\
Gender & $14 / 11$ & $15 / 14$ & $12 / 9$ & $14 / 8$ \\
$($ female $/$ male $)$ & & & & \\
BMI $\left(\mathrm{kg} / \mathrm{m}^{2}\right)$ & $31.70(30.20,37.90)$ & $25.00(22.65,26.75)$ & $31.60(29.65,36.00)$ & $25.60(22.60,27.28)$ \\
\hline
\end{tabular}

\section{Gastroesophageal reflux symptoms from GerdQ}

According to the items of GerdQ symptom score scale (including heartburn, reflux, epigastric pain, nausea, sleep disturbance caused by reflux heartburn, taking 
additional medication), the GerdQ score and gastroesophageal reflux symptoms of O-G group and NO-G group were analyzed. The results show that:

\section{GerdQ score}

The GerdQ score of O-G group [8.00 (7.00,9.00)] was significantly lower than that of NO-G group $[9.00(8.00,10.00)](P<0.05)($ Fig. 3)

Gastroesophageal reflux symptoms

In O-G group, the number of patients with heartburn, reflux, epigastric pain, nausea, sleep disturbance caused by reflux heartburn, taking additional medication symptoms was $13,21,19,14,8,8$, accounting for $52.0 \%, 84.0 \%, 76.0 \%, 56.0 \%$, $32.0 \%, 32.0 \%$ of the total; In NO-G group, the number of patients with heartburn, reflux, epigastric pain, nausea, sleep disturbance caused by reflux heartburn, taking additional medication symptoms was $20,22,13,8,9,17$, accounting for $69.0 \%$, $75.9 \%, 44.8 \%, 27.6 \%, 31.0 \%, 58.6 \%$ of the total. If the ratio of the number of people with a certain symptom to the total number of patients in a certain group is greater than $50 \%$, the symptom is regarded as the main symptom. After sorting and analyzing the six symptoms according to the proportion, the main symptoms of the O-G group were: reflux, epigastric pain, nausea, and heartburn, among which "reflux" and "epigastric pain" were the first and second symptoms in O-G group. The main symptoms of NO-G patients were: reflux, heartburn and taking 
additional medication, in which "reflux" and "heartburn" were the first and second symptoms in NO-G group (Fig. 4).

\section{Esophageal pressure results from HRM}

Images of esophageal pressure measurements

Typical images of esophageal motility measurements during a wet swallow in 4 different groups of patients are presented (Fig. 5). The top and bottom pressure bands reflect the UES and LES pressures before, during and after a wet swallow, respectively. The pressure trajectory between UES and LES reflects the changes in esophageal body pressure during the swallow.

Analysis of LES resting pressure

The LES resting pressure of the $O-G$ group $[13.00(7.50,15.00)]$ was significantly lower than that of the NO-NG group $[25.50(22.75,29.00)]$, the NO-G group $[16.00(11.00,20.00)]$, and the O-NG group $[20.00(18.00,24.00)](P<0.05)$; the LES resting pressure of the NO-G group was significantly lower than that of the NO-NG group and the O-NG group $(P<0.05)$; the LES resting pressure of the ONG group was significantly lower than that of the NO-NG group $(P<0.05)$ (Fig. $6 \mathrm{~A})$.

Analysis of EGJ separation distance 
The EGJ separation distance of the O-G group was significantly larger than that of the NO-NG group $[0.00(0.00,0.00)]$, the NO-G group $[0.00(0.00,1.15)]$ and the O-NG group $[0.00(0.00,0.85)](P<0.05)$; the EGJ separation distance of the NO-G group was significantly larger than that of the NO-NG group $(P<0.05)$; there was no statistical difference in the EGJ separation distance between the O-NG group and NO-G group, so do the O-NG group and the NO-NG group either $(P>0.05)$.

Analysis of esophageal length

The esophageal length of the O-G group $[24.30(22.29,26.51)]$ was significantly shorter than that of the NO-NG group [27.97 (27.20,29.08)], the NO-G group [27.68 $(25.59,29.36)]$, and the O-NG group [26.74 $(25.93,28.81)](P<0.05)$; there was no statistical difference in the esophageal length among the O-NG group, the NO-G group and the NO-NG group $(P>0.05)$ (Fig. 6C).

Analysis of LES length

There was no statistical difference in the LES length among the O-G group (2.60 \pm 0.62$)$, the NO-NG group (2.63 \pm 0.68$)$, the NO-G group (2.62 \pm 0.68$)$, and the O-NG group (2.89 \pm 0.68$)(P>0.05)$ (Fig. 6D).

Analysis of DCI

The DCI of the O-G group [833.00 (355.90, 1947.00)] was significantly lower than that of the O-NG group $[1959.00(1385.00,2801.00)](P<0.05)$, there was no 
statistical difference in the DCI of the O-G group compared with the NO-G group [710.50 (581.40),1332.00]] and the NO-NG group [1721.00 (641.90, 2421.00)] $(P>0.05)$; the DCI of the NO-G group was significantly lower than that of the NONG group, the O-NG group $(P<0.05)$; There was no statistical difference in the DCI between the O-NG group and the NO-NG group $(P>0.05)$ (Fig. 6E).

\section{Correlation analysis of gastroesophageal reflux symptoms and esophageal pressure results from HRM}

In order to further understand whether the occurrence of the main gastroesophageal reflux symptoms in the O-G group and the NO-G group correlated to the abnormal esophageal manometry results, the correlation analysis of the ratio of the main symptoms of the two groups and the abnormal esophageal manometry results was carried out. The results were obtained: in the O-G group, there was a negative correlation between the "reflux" symptom and the LES resting pressure, while there was a positive correlation between the "reflux" symptom and the EGJ separation distance $(P<0.05)$; there was no correlation among other results in the O-G group $(P>0.05)$ (Table 2). In the NO-G group, there was a negative correlation between the "reflux" symptom and the DCI $(P<0.05)$; there was no correlation among other results in the NO-G group $(P>0.05)$ (Table 3$)$. 
TABLE 2. Correlation analysis of main gastroesophageal reflux symptoms and esophageal pressure measurement in obese GERD group

\begin{tabular}{lrrrrrr}
\hline \multirow{2}{*}{ Item } & \multicolumn{2}{l}{ LES resting pressure } & \multicolumn{2}{l}{ EGJ separation distance } & \multicolumn{2}{l}{ esophageal length } \\
\cline { 2 - 7 } & R value & P value & r value & P value & r value & P value \\
\hline Reflux & -0.404 & 0.045 & 0.528 & 0.007 & 0.091 & 0.666 \\
Upper abdominal pain & -0.190 & 0.364 & 0.269 & 0.193 & 0.052 & 0.805 \\
Nausea & 0.0169 & 0.936 & 0.006 & 0.979 & -0.296 & 0.150 \\
Heartburn & -0.213 & 0.308 & 0.388 & 0.056 & 0.056 & 0.792
\end{tabular}

TABLE 3. Correlation analysis of gastroesophageal reflux symptoms and abnormal highresolution esophageal pressure measurement in non-obese GERD group

\begin{tabular}{llccccc}
\hline \multirow{2}{*}{ Item } & \multicolumn{2}{l}{ LES resting pressure } & \multicolumn{2}{l}{ EGJ separation distance } & \multicolumn{2}{c}{ DCI } \\
\cline { 2 - 6 } & R value & P value & R value & P value & R value & P value \\
\hline Reflux & 0.092 & 0.636 & 0.078 & 0.688 & -0.453 & 0.014 \\
Heartburn & 0.228 & 0.235 & 0.005 & 0.980 & -0.321 & 0.090 \\
Meditation & 0.118 & 0.544 & 0.054 & 0.780 & -0.159 & 0.410 \\
\hline
\end{tabular}

Although the "reflux" symptom is the main symptom common with the obese and non-obese GERD patients, there were differences in the esophageal manometry results correlated to the appearance of symptoms between the two groups. 


\section{DISCUSSION}

The main finding of this study is that compared with the non-obese GERD group, the obese GERD group has different gastroesophageal reflux symptoms and esophageal motility characteristics. The obese GERD patients have a lower GerdQ score than the non-obese GERD patients. More than $50 \%$ of the patients in the obese GERD group had symptoms of reflux, epigastric pain, nausea, and heartburn, while more than $50 \%$ of the patients in the non-obese GERD group had symptoms of reflux, heartburn, and taking additional medication. Compared with the nonobese GERD patients, the obese GERD patients have lower LES resting pressure, bigger EGJ separation distance and shorter esophageal length. The obese GERD patients have different symptom-correlated esophageal motility mechanisms: in the obese GERD group, patients with reduced LES resting pressure or increased EGJ separation distance are more likely to have "reflux" symptoms. In the non-obese GERD group, patients with reduced DCI were more likely to have "reflux" symptoms; the two groups of patients had different esophageal dynamic factors related to the appearance of the "reflux" symptoms.

In the past thirty years, the prevalence of obesity worldwide has been increasing at an alarming rate (from $15 \%$ to $32.9 \%$ ). ${ }^{21}$ The onset of obesity is a relatively complex process, which is the result of the combined effects of

physiological, economic, behavioral, genetic, environmental and social factors. ${ }^{22,23}$ 
Although BMI is not the most accurate measure of obesity, it is the most commonly used index to measure obesity. ${ }^{24}$ The onset of a variety of metabolicrelated complications such as acute myocardial infarction, non-alcoholic fatty liver, hypertension, type 2 diabetes, sleep apnea syndrome, etc. is very common in patients with obesity. ${ }^{25}$ As the prevalence of both is increasing year by year, many researchers have linked the incidence of GERD with obesity recently. ${ }^{26,27}$ Many researches have shown that there are multiple mechanisms that may increase the incidence of reflux in overweight or obese people, including: mechanical factors (additional pressure below the Gastroesophageal Junction causing by abdominal/visceral obesity), behavioral factors (smoking, drinking, drugs, high-fat diet, etc.), transient relaxation of LES, and biochemical factors (saliva bicarbonate secretion) are all causes of reflux. ${ }^{28}$ The relationship between obesity and GERD is currently ambiguous, and the specific mechanism is not very clear. There are few reports about the gastroesophageal reflux symptoms and esophageal dynamics in GERD patients with obesity. In this study, we analyzed the difference between the GerdQ score, gastroesophageal reflux symptoms, and HRM test results of the obese GERD patients and non-obese GERD patients, and analyze the correlation of symptoms and HRM test result, to explore the symptoms and esophageal dynamics characteristics of obese GERD patients, as well as the relationship between reflux symptoms and esophageal motility indicators. 
The GerdQ score is currently one of the common diagnostic methods for GERD. In this study, patients who have been clearly diagnosed with GERD were divided into obese and non-obese groups. By Comparing the GerdQ score of the two GERD groups showed that the GerdQ score of the obese GERD group was significantly lower than that of the non-obese GERD group. It is suggested that the severity of gastroesophageal reflux in the obese GERD group is lower than that in the non-obese GERD group. Main symptom is statistically calculated according to the occurrence of each type of gastroesophageal reflux symptoms (heartburn, reflux, epigastric pain, nausea, sleep disorders caused by reflux heartburn, whether to take additional medication). If the ratio of the number of people with a certain symptom to the total number of patients in a certain group is greater than $50 \%$, the symptom is regarded as the main symptom. According to the proportion of the six symptoms, the main symptoms of the obese GERD group were reflux, epigastric pain, nausea and heartburn, while those of the non-obese GERD group were reflux, heartburn and taking additional medication. The results showed that the main symptoms of the obese GERD group were different from those of the non-obese GERD group, and the symptoms of the obese GERD group were less typical. Some literature shows that, compared with the western countries, the incidence of GERD in Asia is mostly mild to moderate, and mainly non-erosive reflux disease (NERD) ${ }^{29,30}$ In this study, it was found that when GERD patients were divided into 
obese and non-obese groups, in addition to "reflux" as the first symptom, "epigastric pain" replaced "heartburn" as the second symptom of the obese GERD patients. The reason for the difference in symptoms between the two groups may be related to the abnormal esophageal motility in obese and non-obese GERD patients. Therefore, in this study, we analyzed the difference of esophageal motility between the two groups, and analyzed the correlation with the occurrence of the gastroesophageal reflux symptoms, in order to clarify the reasons for the different frequency of symptoms between the obese GERD group and non-obese GERD group.

HRM is currently the gold standard for evaluating esophageal motor function. HRM's revolutionary technology makes it possible for doctors to observe and measure changes in pressure throughout the esophagus (that is, from UES to LES). ${ }^{31}$ In 2009, in order to cope with the rapid development of diagnosis and treatment of GERD, a classification method of esophageal motility disorder, The Chicago Classification, was developed for HRM. The updated version 3.0 is now widely used in clinic. ${ }^{20,32}$ In previous studies, the effect of obesity on esophageal motility is contradictory and controversial. Therefore, in this study, we compared the esophageal morphological parameters, esophageal body peristalsis parameters and EGJ parameters between obese GERD patients, non-obese GERD patients, obese non-GERD patients, and non-obese non-GERD patients through the method 
of detailed grouping and comparison, in order to further analyze the effect of obesity on esophageal motility.

The comparison of LES resting pressure among the four groups showed that LES resting pressure of obese GERD group $<$ non-obese GERD group $<$ obese nonGERD group $<$ non-obese non-GERD group. It means that the strength of esophageal anti-reflux barrier of obese GERD group $<$ non-obese GERD group $<$ obese non-GERD group $<$ non-obese non-GERD group, among which the obese GERD group had the weakest esophageal anti-reflux barrier. The decrease of LES resting pressure can make the gastric contents flow into the esophagus more easily, and the decrease of LES resting pressure in the obese GERD patients is more serious, indicating that patients with obesity are more likely to develop GERD. This also supports the view of other researchers that the obese GERD patients have LES defects compared with the normal-weight GERD patients. Therefore, the decrease of LES resting pressure is related to the occurrence of GERD in patients with obesity. In addition, from the results that the LES resting pressure of the obese non-GERD group is higher than that of the non-obese GERD group but lower than that of the non-obese non-GERD group, it can be concluded that there is also a decrease of LES resting pressure in obese non-GERD patients, although it has not reached the level of the GERD patients, but the esophageal anti-reflux 
barrier of obese non-GERD patients is weaker than that of the normal weight patients, and it is easier to further develop to GERD.

The EGJ is composed of CD and LES. EGJ morphology is an important determinant of EGJ barrier function, and spatial separation of EGJ can promote the occurrence of GERD. ${ }^{33}$ One study found that the separation distance of EGJ is related to reflux. The greater the separation distance, the higher the probability of reflux. $^{34}$ The results of our study showed that the results of EGJ segregation distance were as follows: obese GERD group > non-obese GERD group > nonobese non-GERD group, obese GERD group $>$ obese non-GERD group. It is suggested that there is abnormal morphology of EGJ in both obese GERD group and non-obese GERD group, especially in obese GERD group. The abnormal morphology of EGJ in obese GERD group and non-obese GERD group can lead to damage of EGJ barrier function and lead to reflux, especially in obese GERD group. No abnormal morphology of EGJ was found in obese non-GERD patients. This suggests that obesity may not be an independent factor leading to abnormal morphology of the EGJ, but a risk factor for the aggravation of GERD. The specific mechanism of the effect of obesity on the abnormal EGJ morphology in patients with GERD needs to be further studied.

The shortening of the length of the esophagus will cause the increased intraabdominal pressure to be unable to be effectively transmitted to the LES, which 
greatly weakens the effectiveness of the esophageal anti-reflux barrier and significantly increases the possibility of gastric contents flowing back into the esophagus, especially when the abdominal pressure increases after meals or when in standing position, which is an important reason for the increase of transient reflux. Some studies have shown that patients with GERD are more likely to have shorter esophagus and elevated esophagus. ${ }^{35,36}$ It was observed that the length of esophagus in obese GERD group was significantly shorter than that in non-obese GERD group, obese non-GERD group and non-obese non-GERD group. There was no significant difference in esophageal length among non-obese GERD group, obese non-GERD group and non-obese non-GERD group. Based on the unique changes of esophageal anatomy in obese patients with GERD, it is proved that obesity is an important reason for the aggravation of GERD. However, the reason for the shortening of esophageal length caused by obesity is anatomical, individual differences, neurogenic, or myogenic, needs to be further studied in depth.

There was no significant difference in LES length among obese GERD group, non-obese non-GERD group, non-obese GERD group and obese nonGERD group. It shows that obesity does not damage the integrity of LES, and the change of LES length is not the cause of GERD.

HRM's assessment of esophageal contractile function was based on the distal contraction score (DCI). It is reported that the stronger the peristalsis of the body 
of the esophagus, the less the probability and degree of dysphagia, and the increase of the intensity of esophageal peristalsis is beneficial to the movement of the food mass. ${ }^{37}$ The results of this study showed that the DCI of obese GERD group was significantly lower than that of obese non-GERD group, and there was no significant difference compared with non-obese GERD group and non-obese nonGERD group; the DCI of non-obese GERD group was significantly lower than that of non-obese non-GERD group and obese non-GERD group; there was no significant difference in DCI between obese non-GERD group and non-obese nonGERD group. The results showed that the esophageal peristaltic function of obese GERD group and non-obese GERD group was weaker than that of obese nonGERD group, and that of non-obese GERD group was weaker than that of nonobese non-GERD group, but there was no significant difference between obese GERD group, non-obese GERD group and non-obese non-GERD group. Because the food clearance is related to esophageal peristalsis, lower DCI means lower esophageal peristalsis, resulting in a series of symptoms such as obstruction, heartburn and acid regurgitation due to food retention in the esophagus. The results of this study suggest that there is no significant difference in esophageal peristaltic function between obese and non-obese GERD patients. Compared with non-obese non-GERD patients, the esophageal peristaltic function of obese non-GERD patients has no significant difference, but by observing the overall trend, it can be 
found that the esophageal peristaltic function of obese non-GERD patients has an enhanced trend. In addition, it is not very clear whether obese GERD patients induce GERD, due to decreased esophageal peristalsis or patients with obesity reduced esophageal peristalsis after GERD, which needs to be further explored.

The results of GerdQ score showed that the severity of gastroesophageal reflux in the obese GERD group was lower than that in the non-obese GERD group. The analysis of esophageal manometry results showed that the degree of esophageal reflux barrier damage in obese GERD group was greater than that in non-obese GERD group. The above contradictory results suggest that the GerdQ score cannot accurately reflect the severity of the obese GERD patients, and it is necessary to comprehensively evaluate the condition of the obese GERD patients combined with HRM and 24-hour esophageal $\mathrm{pH}$ monitoring in order to formulate a more reasonable treatment plan.

The main symptoms of obese GERD patients were reflux, epigastric pain, nausea and heartburn. The main symptoms of non-obese GERD patients were reflux, heartburn and additional medication. Compared with the results of esophageal manometry in non-obese non-GERD group, the indexes that were different in the obese GERD group were LES resting pressure, EGJ separation distance and esophageal length, while those in the non-obese GERD group were LES resting pressure, EGJ separation distance and DCI. In order to further 
understand the correlation between the main symptoms and abnormal esophageal manometry in the obese GERD group and non-obese GERD group, through the correlation analysis between the ratio of main symptoms of patients with main symptoms and the abnormal results of esophageal manometry, the results showed that there was a negative correlation between the occurrence of "reflux" symptoms and LES resting pressure in obese GERD group, that is, the lower the LES resting pressure, the more likely it is to have "reflux" symptoms, which has a positive correlation with the EGJ separation distance, that is, the larger the EGJ separation distance, the more likely it is to appear "reflux" symptoms. There was a negative correlation between the occurrence of "reflux" symptoms and DCI in non-obese GERD group, that is, the lower the DCI, the more likely they were to have "reflux" symptoms. It is suggested that patients with lower LES pressure or larger separation distance of EGJ in obese GERD group are more likely to have "reflux". In the non-obese GERD group, patients with decreased esophageal peristaltic function were more likely to have "reflux". The above results suggest that there are differences in the influencing factors of "reflux" symptoms between obese and non-obese GERD patients. For the atypical symptom of "epigastric pain", which has the second highest proportion in the obese GERD group, no correlation was found between the atypical symptom and the abnormal index of esophageal manometry in this experiment. Therefore, in addition to esophageal motility 
disorders, we should also consider the effects of gastric motility disorders or abnormal intra-gastric pressure on the occurrence and symptoms of GERD in patients with obesity, to further clarify the symptomatic characteristics and pathogenesis of GERD patients with obesity.

Therefore, it is not difficult to conclude that the changes in esophageal dynamics are different between obese and non-obese GERD patients. Our results show that the obese GERD patients have abnormal esophageal motility, such as damaged esophageal barrier, abnormal morphology of EGJ, abnormal anatomical structure of esophagus and so on. The occurrence of "reflux" symptoms in obese GERD patients was negatively correlated with LES resting pressure and positively correlated with EGJ separation distance, which was different from that in nonobese GERD patients. However, the atypical symptom of epigastric pain in obese GERD patients was not found to be correlated with abnormal esophageal manometry in this experiment. Therefore, we infer that the effect of obesity on the incidence of GERD is not a single factor of abnormal esophageal motility, and the effects of abnormal gastric emptying and intra-gastric pressure on the pathogenesis of GERD should be further discussed. Long-term esophageal pressure measurement and 24-hour esophageal $\mathrm{pH}$ monitoring will help to further explain the mechanism of GERD in people with obesity. In the next experimental stage, we need to increase the waist-to-height ratio, fatty liver, abdominal circumference, 
blood lipids, biochemical tests, Electrogastrogram, gastrointestinal ultrasound and other tests to further examine and explore the impact of gastric dysfunction on the occurrence of reflux in obese GERD patients.

To sum up, the effects of obesity on esophageal motility in GERD patients are diverse, suggesting that the pathophysiological changes of obese GERD patients may be different from those of non-obese GERD patients. Different treatment methods should be adopted according to specific pathophysiological factors in the treatment of obesity-related GERD. To understand the effect of obesity on esophageal motility in patients with GERD, we can choose a more suitable scheme for the treatment of obese GERD patients. Because some GERD patients with morbidly obesity will choose anti-reflux surgery combined with weight loss/bariatric surgery for treatment when it's difficult to achieve a good effect with use of meditation. Patients are examined by HRM before operation to understand the differential effect of obesity on esophageal motility in GERD, which can provide more reliable basis for the choice of operation. At the same time, it also provides some reference for patients with obesity who want to undergo weight loss surgery in the prevention and treatment of surgery-related GERD complications.

This experiment is a retrospective analysis, the experiment is a single center study, the sample size is small, and the number of cases in each group is small. In 
the next step, the number of cases will be expanded, and the results of 24-hour $\mathrm{pH}-$ impedance examination, electrogastrogram and gastrointestinal ultrasound will be included to study the esophageal motility and acid reflux time, gastric emptying and intra-gastric pressure in obese patients. In addition, all the cases came from our hospital, which may cause limitations and regional differences, so it is necessary to carry out multi-center and large sample statistics. With the continuous improvement of the research level and the in-depth study of obese GERD patients, it will play a positive guiding role in the diagnosis, treatment and prognosis of such patients.

\section{CONCLUSIONS}

Comparing with GERD patients without obesity, the symptoms, esophageal motility, esophageal motility mechanism related to the symptoms of GERD patients with obesity got different characteristics. This result provides a reference for treatment options of GERD patients with obesity.

\section{ABBREVIATIONS}

GERD: Gastroesophageal reflux disease; BMI: Body Mass Index; EE: Erosive Esophagitis; BE: Barrett's Esophagus; DCI: Distal esophageal contraction integral; MII: Multichannel Intraluminal Impedance; HRM: High Resolution Manometry; LES: Lower esophageal sphincter; EGJ: esophagus-gastric junction; LESP: lower 
esophageal sphincter resting pressure; PPIs: Proton pump inhibitors; CD: crural diaphragm; UES: Upper esophageal sphincter; WHO: the World Health Organization; DALY: Disability-Adjusted Life-Years.

\section{DECLARATIONS}

\section{Ethics approval and consent to participate}

The Ethics Committee of First Affiliated Hospital of Dalian Medical University

Ethical approved the report of scientific research project. It confirmed that project program and informed consent were reviewed. All the human subjects in the study had signed the informed consent. (Approval number: PJ-KS-KY-2018-69(X)).

\section{Consent for publication}

Not applicable.

\section{Availability of data and materials}

All relevant data are included within the paper and their supporting information files. Raw Data are available from the corresponding author for researchers who meet the criteria for access to confidential data.

\section{Competing interests}

The authors declare that they have no competing interests. 


\section{Funding}

The authors received no specific funding for this work.

\section{Authors' contributions}

Study concept and design: YY, ZD, AR. Acquisition of data, analysis and interpretation of data: YY, LM, DY, ZZ, ZD, SJ. Drafting of the manuscript: YY, AR LM, DY, ZZ, ZD. Critical revision of the manuscript for important intellectual content: YY, PZ, LS, ZD, AR. Statistical analysis: YY. Administrative, technical, or material support: YY, PZ, LS, ZD, LM, DY, ZZ, SJ. Study supervision: ZD, SJ. All authors read and approved the final manuscript.

\section{Acknowledgements}

We are indebted to all related departments of our hospital for their encouraging criticism and discussions.

\section{REFERENCES}

1. Hungin APS, Molloy-Bland M, Scarpignato C. Revisiting Montreal: New Insights into Symptoms and Their Causes, and Implications for the Future of GERD. Am J Gastroenterol. 2019;114:414-21.

2. Zvenyach T, Pickering MK. Health Care Quality: Measuring Obesity in Performance Frameworks. Obesity (Silver Spring). 2017;25:1305-12. 
3. Kim TJ, Lee H, Baek SY, Kim K, Min YW, Min BH, et al. Metabolically Healthy Obesity and the Risk of Erosive Esophagitis: A Cohort Study. Clin Transl Gastroenterol. 2019;10(9):e00077.

4. Khoma O, Burton L, Falk MG, Van der Wall H, Falk GL. Predictors of reflux aspiration and laryngo-pharyngeal reflux. Esophagus. 2020;17:355-62.

5. Ng M, Fleming T, Robinson M, Thomson B, Graetz N, Margono C, et al. Global, regional, and national prevalence of overweight and obesity in children and adults during 1980-2013: a systematic analysis for the Global Burden of Disease Study 2013 Lancet. 2014;384:766-81.

6. World Health Organization (2020). Obesity and Overweight Fact Sheet. April 1, 2020. Available at: https://www.who.int/news-room/fact-sheets/detail/obesity-andoverweight. Accessed June 26, 2020.

7. NCD Risk Factor Collaboration. Trends in adult body-mass index in 200 countries from 1975 to 2014: a pooled analysis of 1698 population-based measurement studies with $19 \cdot 2$ million participants Lancet. 2016;387:1377-96.

8. Zhou B. Predictive values of body mass index and waist circumference for risk factors of certain related diseases in Chinese adults - study on optimal cut-off points of body mass index and waist circumference in Chinese adults. Biomedical and environmental sciences: BES. 2002;15:83-96. 
9. Williams EP, Mesidor M, Winters K, Dubbert PM, Wyatt SB. Overweight and Obesity: Prevalence, Consequences, and Causes of a Growing Public Health Problem. Curr Obes Rep. 2015;4:363-70.

10. Vaishnav B, Bamanikar A, Maske P, Reddy A, Dasgupta S. Gastroesophageal Reflux Disease and its Association with Body Mass Index: Clinical and Endoscopic Study. J Clin Diagn Res. 2017;11:OC01-OC04.

11. Jacobson BC, Somers SC, Fuchs CS, Kelly CP, Camargo CA Jr. Body-mass index and symptoms of gastroesophageal reflux in women. $\mathrm{N}$ Engl J Med. $2006 ; 354: 2340-8$.

12. Blevins CH, Dierkhising RA, Geno DM, Johnson ML, Vela MF, Ravi K, et al. Obesity and GERD impair esophageal epithelial permeability through 2 distinct mechanisms. Neurogastroenterol Motil. 2018;30:e13403.

13. Lee SW, Lien HC, Lee TY, Tung CF, Yeh HZ, Chang CS. Impact of Obesity on a Chinese Population with Erosive Esophagitis and Barrett's Esophagus. Gut Liver. 2017;11:377-82.

14. Qumseya B, Gendy S, Wallace A, Yang D, Estores D, Ayzengart A, et al. Prevalence of Barrett's esophagus in obese patients undergoing pre-bariatric surgery evaluation: a systematic review and meta-analysis. Endoscopy. 2020;52:537-47. 
15. Ashrafi D, Osland E, Memon MA. Bariatric surgery and gastroesophageal reflux disease. Ann Trans1 Med. 2020;8:S11.

16. Ayazi S, Hagen JA, Chan LS, DeMeester SR, Lin MW, Ayazi A, et al. Obesity and gastroesophageal reflux: quantifying the association between body mass index, esophageal acid exposure, and lower esophageal sphincter status in a large series of patients with reflux symptoms. J Gastrointest Surg. 2009;13:1440-7.

17. Valezi AC, Herbella FAM, Schlottmann F, Patti MG. Gastroesophageal Reflux Disease in Obese Patients. J Laparoendosc Adv Surg Tech A. 2018;28:949-52.

18. Gyawali CP, Kahrilas PJ, Savarino E, Zerbib F, Mion F, Smout AJPM, et al. Modern diagnosis of GERD: the Lyon Consensus. Gut. 2018;67:1351-62.

19. Zhou WC, Jia L, Chen DY, Liu Y, Liu J, Jiang SM, et al. The effects of paroxetine and amitriptyline on the upper esophageal sphincter (UES) pressure and its natural history in globus pharyngeus. Dig Liver Dis. 2017;49:757-63.

20. Kahrilas PJ, Bredenoord AJ, Fox M, Gyawali CP, Roman S, Smout AJ, et al. The Chicago Classification of esophageal motility disorders, v3.0. Neurogastroenterol Motil. 2015;27:160-74.

21. Nguyen DM, El-Serag HB. The epidemiology of obesity. Gastroenterol Clin North Am. 2010;39:1-7.

22. Sellayah D, Cagampang FR, Cox RD. On the evolutionary origins of obesity: a new hypothesis. Endocrinology. 2014;155:1573-88. 
23. Bhupathiraju SN, Hu FB. Epidemiology of Obesity and Diabetes and Their Cardiovascular Complications. Circ Res. 2016;118:1723-35.

24. Batsis JA, Mackenzie TA, Bartels SJ, Sahakyan KR, Somers VK, LopezJimenez F. Diagnostic accuracy of body mass index to identify obesity in older adults: NHANES 1999-2004. Int J Obes (Lond). 2016;40:761-7.

25. Apovian CM. Obesity: definition, comorbidities, causes, and burden. Am J Manag Care. 2016;22:176-85.

26. Nadaleto BF, Herbella FA, Patti MG. Gastroesophageal reflux disease in the obese: Pathophysiology and treatment. Surgery. 2016;159:475-86.

27. Kristo I, Paireder M, Jomrich G, Felsenreich DM, Nikolic M, Langer FB, et al. Modern Esophageal Function Testing and Gastroesophageal Reflux Disease in Morbidly Obese Patients. Obes Surg. 2019;29:3536-41.

28. Gorodner V, Viscido G, Signorini F, Obeide L, Moser F. Gastroesophageal reflux disease and morbid obesity: evaluation and treatment. Updates Surgery, 2018, 70(3):331-7.

29. Sharma N, Hui T, Wong HC, Srivastava S, Teh M, Yeoh KG, et al. Risk stratifying the screening of Barrett's esophagus: An Asian perspective. JGH Open. 2017;1:68-73. 
30. Takahashi K, Seki Y, Kasama K, Amiki M, Baba S, Ito M, et al. Prevalence of reflux esophagitis in obese Japanese undergoing bariatric surgery. JGH Open. 2019;4:519-24.

31. Keller J. What Is the Impact of High-Resolution Manometry in the Functional Diagnostic Workup of Gastroesophageal Reflux Disease?. Visc Med. 2018;34:1018.

32. Patel A, Cassell B, Sainani N, Wang D, Shahid B, Bennett M, et al. Comparison of motor diagnoses by Chicago Classification versions 2.0 and 3.0 on esophageal high-resolution manometry. Neurogastroenterol Motil. 2017;29:10.1111/nmo.13042.

33. Ding H, Duan Z, Yang D, Zhang Z, Wang L, Sun X, et al. High-resolution manometry in patients with and without globus pharyngeus and/or symptoms of laryngopharyngeal reflux. BMC Gastroenterol. 2017;17:109.

34. Tolone S, de Cassan C, de Bortoli N, Roman S, Galeazzi F, Salvador R, et al. Esophagogastric junction morphology is associated with a positive impedance-pH monitoring in patients with GERD. Neurogastroenterol Motil. 2015;27:1175-82. 35. Ness-Jensen E, Hveem K, El-Serag H, Lagergren J. Lifestyle Intervention in Gastroesophageal Reflux Disease. Clin Gastroenterol Hepatol. 2016;14:175-82. 
36. Tanaka Y, Ihara E, Nakamura K, Muta K, Fukaura K, Mukai K, et al. Clinical characteristics associated with esophageal motility function. J Gastroenterol Hepatol. 2016;31:1133-40.

37. Liu L, Li S, Zhu K, Yu W, Wang H, Guo J, et al. Relationship between esophageal motility and severity of gastroesophageal reflux disease according to the Los Angeles classification. Medicine (Baltimore). 2019;98:e15543.

\section{FIGURE LEGENDS}

FIGURE 1. Manometer of normal esophagus. A: UES and LES resting state without swallowing; B: Image of normal esophageal contraction when swallowed with warm water.

FIGURE 2. Measurement of the separation distance between the LES and CD under HRM.

FIGURE 3. Comparison of GerdQ scores in different groups. O-G: obese GERD group; NO-G: non-obese GERD group.

FIGURE 4. The composition ratio of gastroesophageal reflux symptoms (heartburn, reflux, epigastric pain, nausea, reflux heartburn caused sleep disorders, whether to take extra meditation) in different groups. O-G: obese GERD group; NO-G: non-obese GERD group. 
FIGURE 5. Typical high-resolution esophageal pressure measurements in different groups. A: obese GERD group; B: non-obese GERD group; C: obese non-GERD group; D: non-obese non-GERD group.

FIGURE 6. Esophageal manometric parameters in different groups. A: The resting pressure of LES in four groups of patients; B: The EGJ separation distance in four groups of patients; C: The esophagus length in four groups of patients; D: The LES length in four groups of patients; E: The DCI length in four groups of patients; OG: obese GERD group; O-NG: obese non-GERD group; NO-G: non-obese GERD group; NO-NG: non-obese non-GERD group. 


\section{Figures}
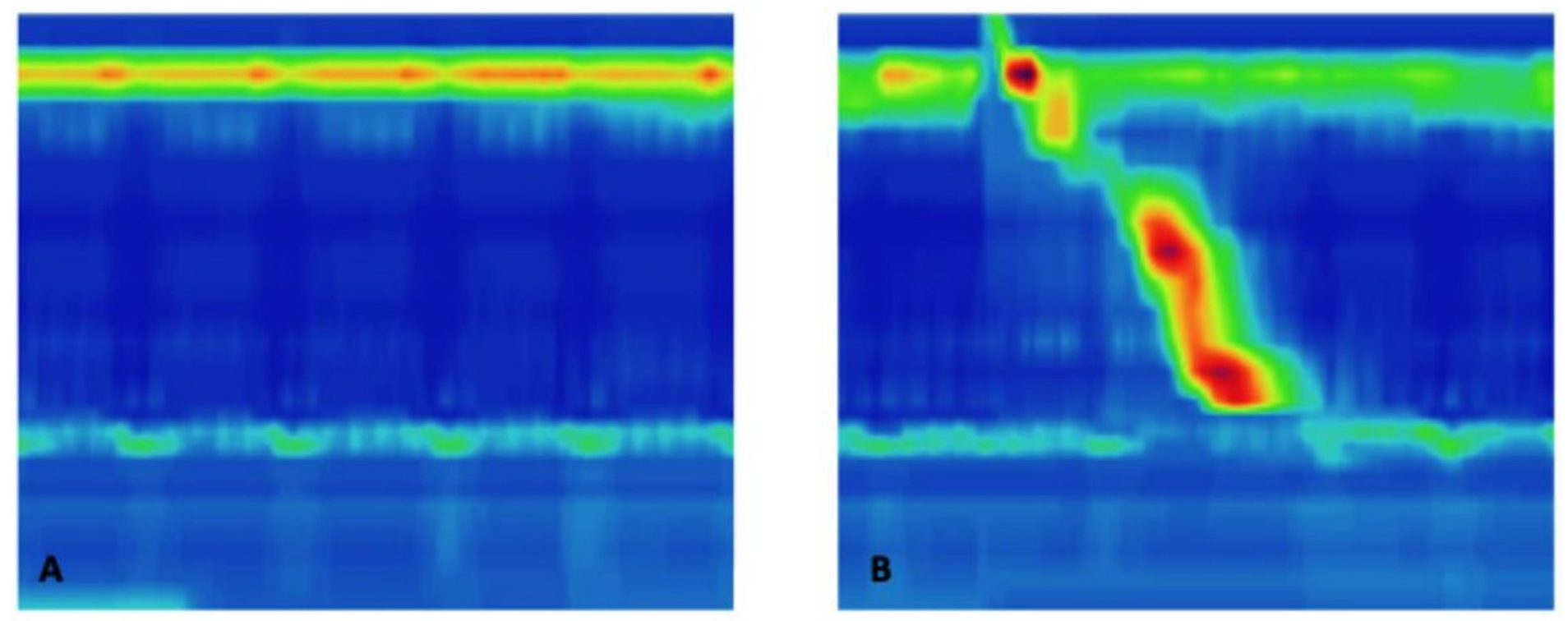

\section{Figure 1}

Manometer of normal esophagus. A: UES and LES resting state without swallowing; B: Image of normal esophageal contraction when swallowed with warm water. 


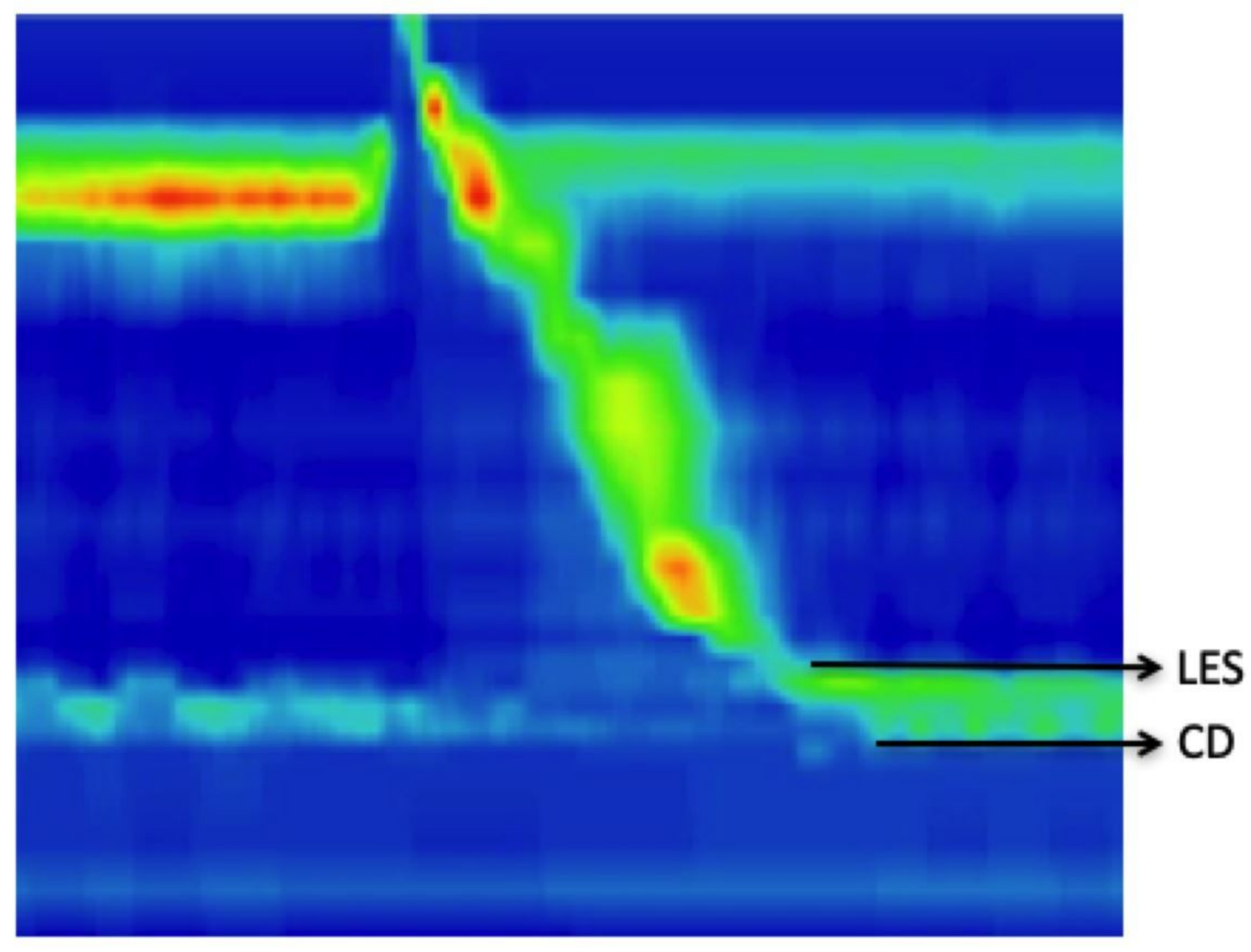

Figure 2

Measurement of the separation distance between the LES and CD under HRM. 


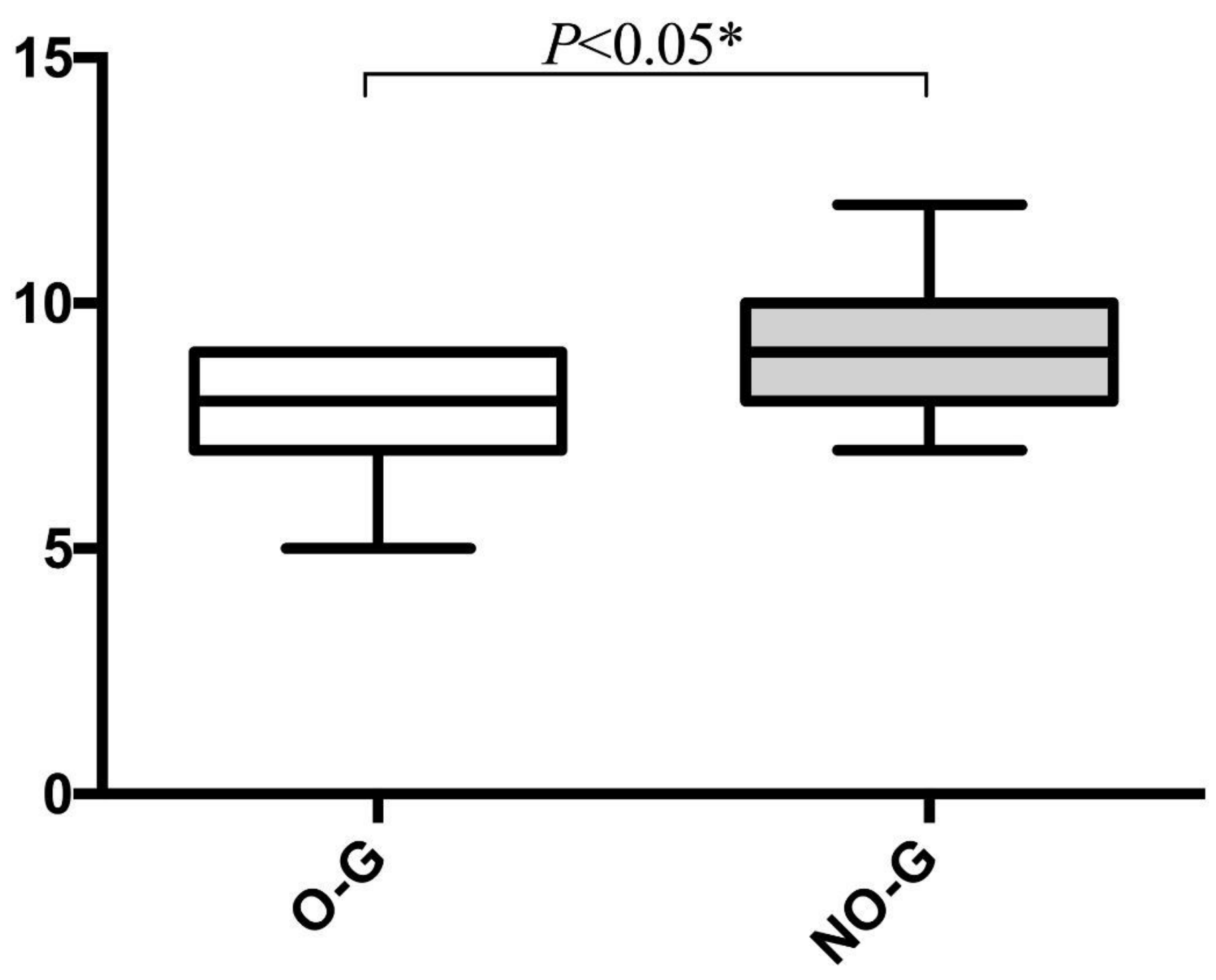

Figure 3

Comparison of GerdQ scores in different groups. O-G: obese GERD group; NO-G: non-obese GERD group. 


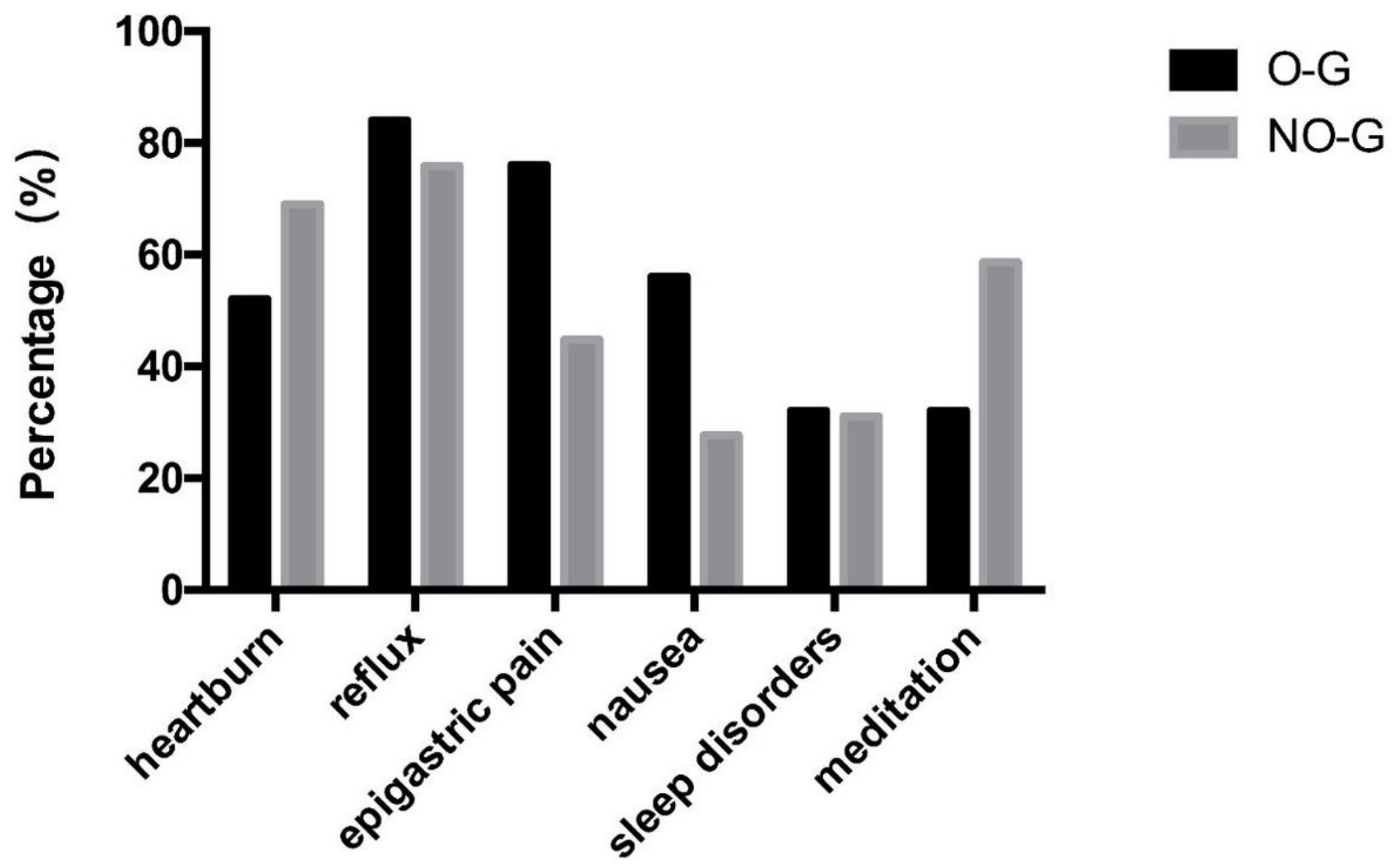

Figure 4

The composition ratio of gastroesophageal reflux symptoms (heartburn, reflux, epigastric pain, nausea, reflux heartburn caused sleep disorders, whether to take extra meditation) in different groups. O-G: obese GERD group; NO-G: non-obese GERD group. 

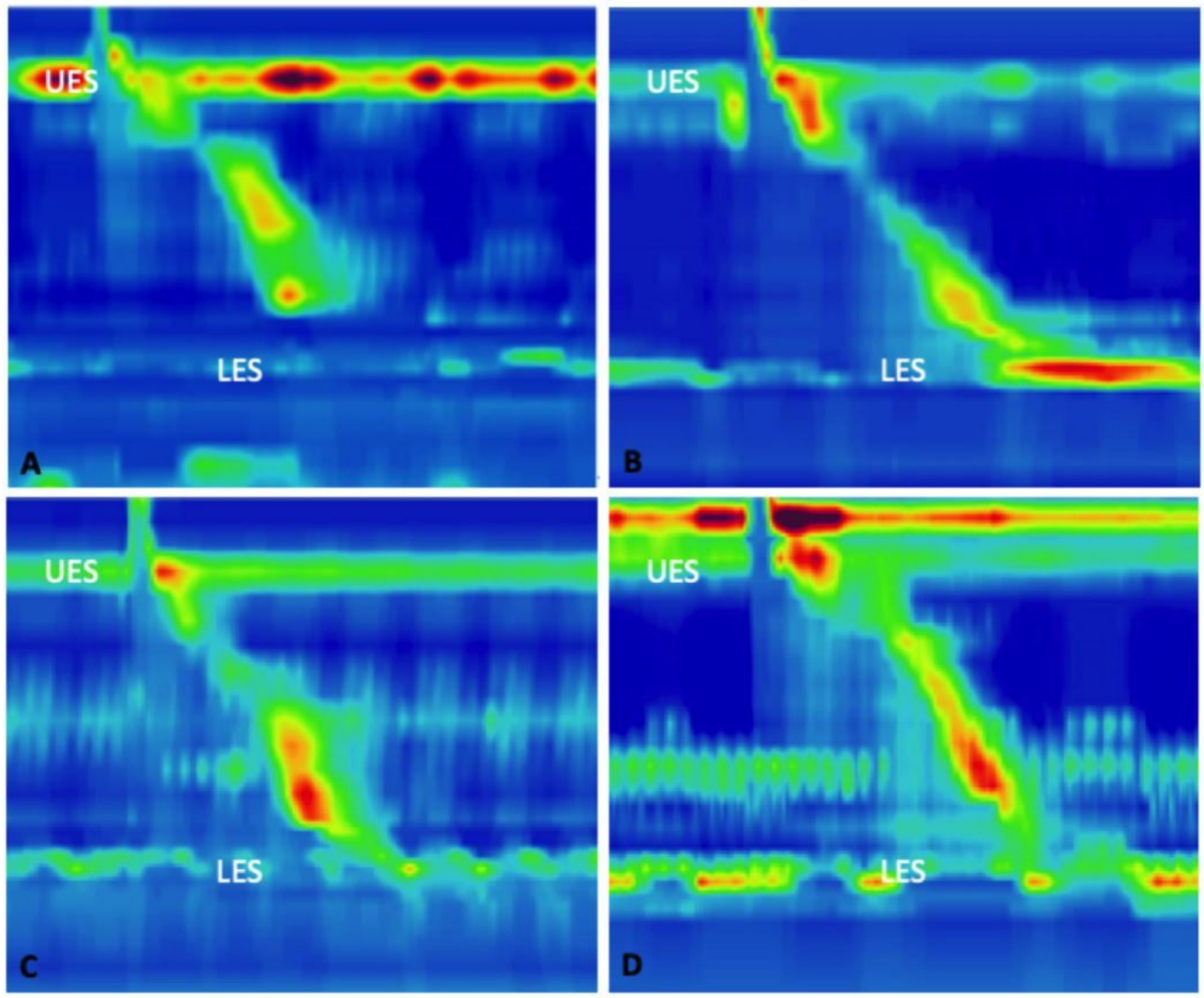

\section{Figure 5}

Typical high-resolution esophageal pressure measurements in different groups. A: obese GERD group; B: non-obese GERD group; C: obese non-GERD group; D: non-obese non-GERD group. 


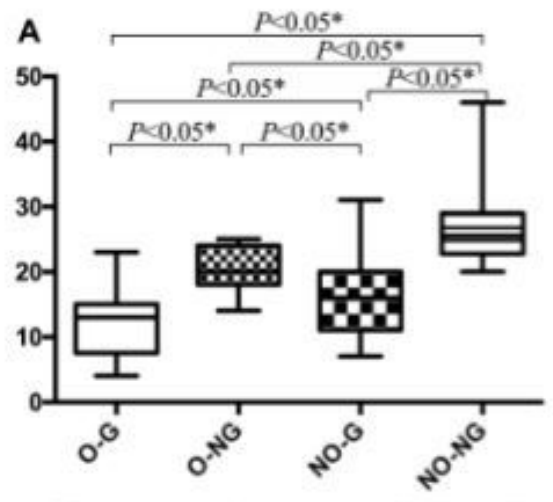

The average resting pressure of $L E S(\mathrm{mmHg})$

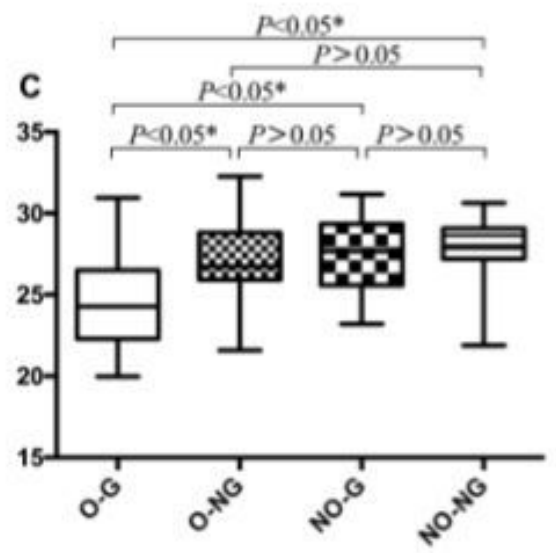

The esophagus length $(\mathrm{cm})$

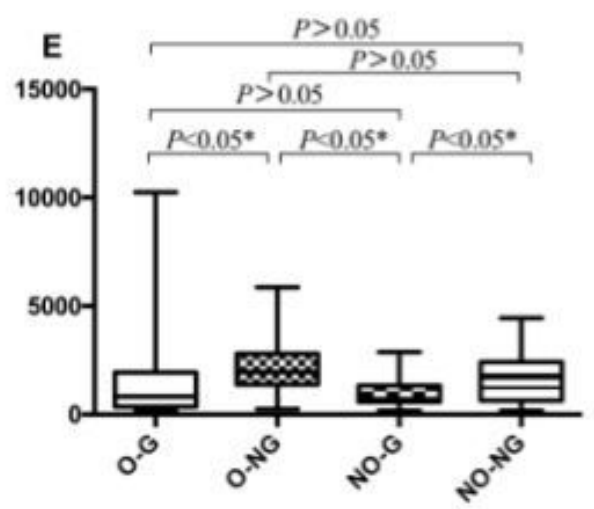

The DCl(mmHg.s.cm)

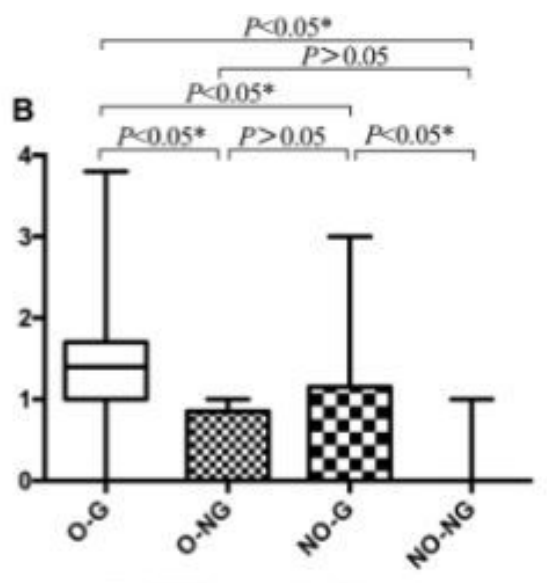

The EGJ separation distance(cm)

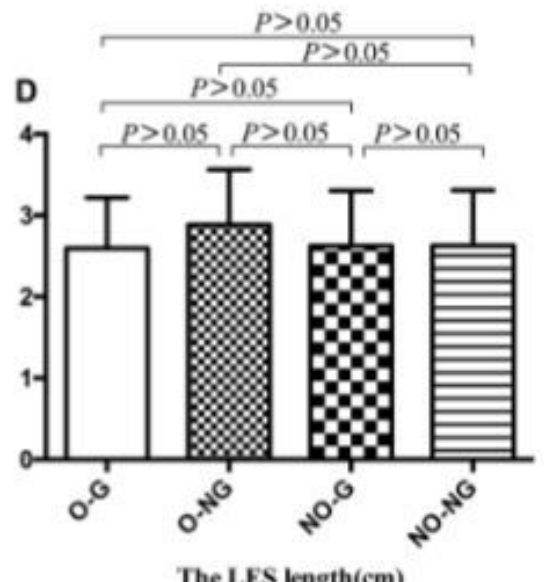

The LES length(cm)

\section{Figure 6}

Esophageal manometric parameters in different groups. A: The resting pressure of LES in four groups of patients; B: The EGJ separation distance in four groups of patients; $C$ : The esophagus length in four groups of patients; D: The LES length in four groups of patients; $E$ : The DCl length in four groups of patients; OG: obese GERD group; O-NG: obese non-GERD group; NO-G: non-obese GERD group; NO-NG: non-obese non-GERD group. 